
\title{
is Research Square \\ Anticoagulation in Patients With a Femoral Neck Fracture: Evaluating Daily Practice.
}

Veronique A.J.I.M. van Rijckevorsel ( $\nabla$ RijckevorselV@maasstadziekenhuis.nl)

Maasstad Ziekenhuis https://orcid.org/0000-0001-5628-6907

Louis de Jong

Franciscus Gasthuis en Vlietland

Taco M.A.L. Klem

Franciscus Gasthuis en Vlietland

Tjallingius M. Kuijper

Maasstad Ziekenhuis

Gert R. Roukema

Maasstad Ziekenhuis

\section{Research article}

Keywords: Hip fracture, hip hemiarthroplasty (HA), coumarin anticoagulants, thrombocyte aggregation inhibitor, clinical outcomes

Posted Date: December 28th, 2020

DOl: https://doi.org/10.21203/rs.3.rs-135707/v1

License: (c) (i) This work is licensed under a Creative Commons Attribution 4.0 International License.

Read Full License 


\section{Abstract}

Background: Geriatric hip fracture patients are fragile and mostly suffering from multiple comorbidities, such as cognitive or cardiovascular disease, in which the use of antiplatelet therapy or vitamin $\mathrm{K}$ antagonists is common. The aim of this study is to assess the effect of antiplatelet therapy and vitamin $\mathrm{K}$ antagonists on perioperative care and patient outcome after hip hemiarthroplasty in daily practice.

Methods: Patients with a femoral neck fracture requiring hip hemiarthroplasty were included in the study. Data were extracted from a prospective hip fracture database and completed by retrospective review of the hospital records at two level II trauma centers between January 1st 2010 and May 16th 2016.

Results: 907 patients (68\% female) were included, with a median age of 84 (IQR 78-88), of which 415 (46\%) used any sort of anticoagulation. Anticoagulation medication was associated with more packed cell supplementation; 0.33 units $( \pm 0.88)$ vs. 0.15 units $( \pm 0.61)(P=<0.001)$. Also, more hematoma's were found for patients with anticoagulation, $19.5 \%$ versus $11.4 \%, P=0.001$. Patients using vitamin $K$ antagonists needed more days to be ready for discharge compared to patients without anticoagulation, 5.5 days (4-9) versus 4 days (3-7), $\mathrm{P}=<0.001$. There was no significant difference in 30-day or 1-year mortality after surgery, nor in time to surgery.

Conclusion: Patients using any sort of anticoagulation are in need of more packed cell supplementation, develop more hematomas and need more days to be ready for discharge, compared to patients without anticoagulation use. Also prolonged time to surgery was found for patients using vitamin $\mathrm{K}$ antagonists.

\section{Background}

One of the reasons for frailty in the hip fracture population is the presence of multiple comorbidities, including a high percentage of cardiovascular disease. ${ }^{(1)}$ Therefore, oral anticoagulants, either vitamin $\mathrm{K}$ antagonists (VKA) or antiplatelet therapy, are used in $40 \%$ of the patients with a hip fracture. ${ }^{(2)}$

Current guidelines advise to perform hip fracture surgery within 24 to 48 hours after admission, minimizing postoperative complications and mortality risk and returning a higher percentage of patients to independent living. ${ }^{(1)(3)(4)}$ The use of VKA, with a natural half-life over 24 hours, is challenging since the use of anticoagulation medication is associated with a higher amount of blood loss during surgery. ${ }^{(5)}$ Therefore, adequate reversal therapy for VKA with either vitamin $\mathrm{K}$ or prothrombin complex concentrate is embedded in the standard of care for patients with a hip fracture and an International Normalized Ratio $\left(\right.$ INR) > 1.5. ${ }^{(1)(5)(6)(7)}$ Current guidelines see no harm in continuing antiplatelet therapy (carbasalate calcium, acetylsalicyclic acid) during the perioperative period, since previous studies show no substantially increased risk for bleeding complications or mortality. ${ }^{(8)(9)(10)(11)}$

A major complication after hip fracture surgery is a deep surgical site infection (SSI), which is partly influenced by the presence of a significant hematoma. ${ }^{(12)}$ The perioperative process to prevent the 
accumulation of a hematoma should therefore be optimal. ${ }^{(1)}$ The aim of this study is therefore to investigate the influence of VKA and antiplatelet therapy on patient outcome such as the presence of a SSI and hematoma after hip hemiarthroplasty.

\section{Methods}

\section{Patient selection}

All patients admitted with a proximal femur fracture between January 1st 2010 and May 16th 2016 were enrolled in this study. Inclusion criteria were a femoral neck fracture for which a hip hemiarthroplasty was placed and an up to date medication lists. All other proximal femur fractures, i.e. intertrochanteric fractures and subtrochanteric fractures, were excluded. (Fig. 1). Also, multitrauma patients were excluded from analysis. Patients underwent surgical treatment in two level II trauma teaching hospitals. Baseline characteristics (age, gender, frailty, time to surgery and type of anesthesia) and clinical outcomes (surgery time, surgical approach, hemoglobin and blood loss, pain, presence of hematoma, reoperation, deep surgical site infection, time needed for discharge and mortality) were obtained from a prospective hip fracture database and were complemented by reviewing hospital records. Follow-up duration was in all patients at least one-year.

\section{Perioperative management}

Perioperative conditions were standardized according to local hospitals protocol. An INR $\leq 1.5$ was required to give spinal anesthesia during surgery. Ten milligrams of vitamin $\mathrm{K}$ was administered to patients with an INR $>1.5$. This was repeated after six hours and repeated if necessary. If surgery was performed shortly after admission, prothrombin complex concentrate was administered. No tranexamic acid or antiplatelet transfusion was used.

\section{Surgical techinque}

All operating theatres had laminar airflow, and all patients received standard antibiotic prophylaxis prior to surgery (1-3 g cefazolin, Kefzol). A cemented (Palamed G, gentamicin impregnated cement; Heraeus, Hanau, Germany) unipolar prosthesis (Mathys CCA; Mathys Ltd. Bettlach, Switzerland) was used in all surgeries. Twenty-eight surgeons performed the operations and determined the desired surgical approach, which was either an (minimally invasive) anterolateral approach or a straight lateral approach.

\section{Assessed parameters}

Blood loss (in $\mathrm{mL}$ ) was noted in the surgical report by either the surgeon or the anesthesiologist. Hemoglobin loss (in $\mathrm{g} / \mathrm{dL}$ ) was calculated as the difference between the preoperative and the lowest postoperative hemoglobin level. To determine the frailty of the included patients, two scores were added. First, the Nottingham Hip Fracture Score (NHFS) was used, in which an NHFS of $\leq 4$ is considered to be a low risk, and an NHFS of $\geq 5$ to be a high risk for 30-day mortality. ${ }^{(13)}$ The second score used was the American Society of Anesthesiologist (ASA) score. The visual analogue scale (VAS) was used to rate the 
pain on the first postoperative day. The days a patient was in actual need of medical therapy and hospital support were defined as days until a patient was ready for discharge. The presence of a hematoma was determined by physical examination, no ultrasound was used.

\section{Statistical analysis}

Data was analyzed using Stata version 14.0 (StataCorp, College Station, Texas). Tests used were the Student's t-test, the Pearson's Chi-squared test, the Wilcoxon rank-sum test, a Kurkskal-Wallis test. In addition linear regression was used to test for differences in continuous outcomes between more than 2 groups.. Categorical variables are presented as frequencies and percentages, and continuous variables are presented as means with a standard deviation (SD) in case of a normal distribution, or as median with an interquartile range (IQR) in case of a skewed distribution. All statistical tests were two-sided with a significance level of $P \leq 0.05$.

For both the baseline characteristics and the univariable analysis, data are presented as both anticoagulation use versus no anticoagulation use, and also for subgroups, in which antiplatelet therapy (AP) (carbasalate calcium and acetylsalicyclic acid) and vitamin $\mathrm{K}$ antagonists (VKA) (acenocoumarol and phenprocoumon) were displayed. Data for clopidogrel (Plavix) were displayed separately, since the population using clopidogrel was too small to test for statistically differences compared to patients using AP or VKA.

\section{Results}

\section{Patient characteristics}

2491 patients were screened for eligibility, of which 907 patients were enrolled in the present study. The inclusion flowchart is presented in Figure A. Table 1. displays the baseline characteristics of the study population. The median age of the population was 84 years (IQR 78-88), 615 were female (68\%), and $56 \%$ had an ASA score $\geq 3$. Subgroup analysis was performed for patients using vitamin $\mathrm{K}$ antagonists and antiplatelet therapy. Both groups were compared to each other and to patients who did not use anticoagulants (Table 2). As seen in Table 2, there are differences between the three groups in all baseline characteristics excluding the variables; preoperatively hemoglobin level, $\mathrm{BMI}$ and type of anesthesia. Three subgroups were compared (AP vs. VKA), (AP vs. no anticoagulation) and (VKA vs. no anticoagulation) to determine differences between anticoagulation types. 
Table 1

Baseline characteristics of the 907 included patients

\begin{tabular}{|c|c|c|c|c|}
\hline Factor & Overall (907) & $\begin{array}{l}\text { No anticoagulation } \\
\text { (492) }\end{array}$ & $\begin{array}{l}\text { Anticoagulation } \\
\text { (415) }\end{array}$ & $\begin{array}{l}\text { P- } \\
\text { value* }\end{array}$ \\
\hline Age (median (IQR) & $84(78-88)$ & $83(76-88)$ & $84(79-88)$ & $0.046^{\rrbracket}$ \\
\hline Female Gender (obs (\%)) & $\begin{array}{l}615 / 907 \\
(67.8)\end{array}$ & $355 / 492(72.2)$ & $260 / 415(62.7)$ & $0.002^{*}$ \\
\hline NHFS (median (IQR)) & $5(4-6)$ & $5(4-5.5)$ & $5(4-5)$ & $0.003^{*}$ \\
\hline ASA score & & & & $0.000 *$ \\
\hline $1-2$ (obs, \%) & $\begin{array}{l}393 / 898 \\
(43.8)\end{array}$ & 263/487 (54.0) & 130/411 (31.6) & \\
\hline 3-4 (obs, \%) & $\begin{array}{l}505 / 898 \\
(56.2)\end{array}$ & $224 / 487(46.0)$ & $281411(68.4)$ & \\
\hline $\begin{array}{l}\text { Preoperatively Hb level (mean } \\
( \pm \text { SD)) }\end{array}$ & $7.98(0.03)$ & $8.01(0.04)$ & $7.93(0.05)$ & $0.179^{\rrbracket}$ \\
\hline $\begin{array}{l}\text { Preoperatively GFR (mean ( } \pm \\
\text { SD)) }\end{array}$ & $66.0(21.6)$ & $69.0(20.0)$ & $62.4(22.9)$ & $0.000^{*}$ \\
\hline BMI & & & & $0.186^{*}$ \\
\hline < 18.5 (obs (\%)) & $38 / 583(6.5)$ & $20 / 287(7.0)$ & 18/296 (6.1) & \\
\hline $18.5-30$ (obs (\%)) & $\begin{array}{l}487 / 583 \\
(83.5)\end{array}$ & 245/287 (85.4) & 242/296 (81.8) & \\
\hline$>30$ (obs $(\%))$ & $\begin{array}{l}58 / 583 \\
(10.0)\end{array}$ & $22 / 287(7.6)$ & $36 / 296(12.1)$ & \\
\hline $\begin{array}{l}\text { Time to surgery (median } \\
\text { (IQR)) }\end{array}$ & $\begin{array}{l}20.4(14.5- \\
26.6)\end{array}$ & $19.6(13.4-24.6)$ & $21.5(15.3-30.6)$ & $0.721^{*}$ \\
\hline Anesthesia & & & & $0.004 *$ \\
\hline Spinal (obs (\%)) & $\begin{array}{l}777 / 902 \\
(86.1)\end{array}$ & $437 / 490(89.2)$ & $340 / 412(82.5)$ & \\
\hline General (obs (\%)) & $\begin{array}{l}125 / 902 \\
(13.9)\end{array}$ & $53 / 490(10.8)$ & $72 / 412(17.5)$ & \\
\hline \multicolumn{5}{|c|}{ Notes: *Pearson’s Chi-squared test, ${ }^{\mathbb{}}$ Student's t-test, ${ }^{¥}$ Wilcoxon rank-sum test } \\
\hline \multicolumn{5}{|c|}{$\begin{array}{l}\text { Abbreviations: NHFS. Nottingham Hip Fracture Score; Hb. Hemoglobin in mmol/L; GFR. glomerular } \\
\text { filtration rate in } \mathrm{ml} / \mathrm{min} / 1.73 \mathrm{~m}^{\wedge} 2 ; \mathrm{ASA} \text {. American Society of Anesthesiology; BMI. Body Mass Index }\end{array}$} \\
\hline \multicolumn{5}{|c|}{ Anticoagulation therapy is vitamin $\mathrm{K}$ antagonists and antiplatelet therapy combined. } \\
\hline
\end{tabular}


Table 2

Baseline characteristics of subgroups

\begin{tabular}{|c|c|c|c|c|}
\hline Factor & $\begin{array}{l}\text { No } \\
\text { anticoagulation } \\
(492)\end{array}$ & $\begin{array}{l}\text { Vitamin k } \\
\text { antagonists (141) }\end{array}$ & $\begin{array}{l}\text { Antiplatelet } \\
\text { therapy (213) }\end{array}$ & $\begin{array}{l}\text { P- } \\
\text { value* }\end{array}$ \\
\hline Age (median (IQR) & $83(76-88)$ & $84(80-88)$ & $84(79-88)$ & $0.034^{\wedge}$ \\
\hline Female Gender (obs (\%)) & $355 / 492(72.2)$ & $92 / 142(64.8)$ & $132 / 213(61.8)$ & $0.017 *$ \\
\hline NHFS (median (IQR)) & $5(4-5.5)$ & $5(4-6)$ & $5(4-6)$ & $0.015^{\prime \prime}$ \\
\hline ASA score & & & & $<.001 *$ \\
\hline $1-2$ (obs, \%) & 263/487 (54.0) & $35 / 141(24.8)$ & 83/211 (39.3) & \\
\hline 3-4 (obs, \%) & 224/487 (46.0) & $106 / 141(75.2)$ & $128 / 211(60.7)$ & \\
\hline $\begin{array}{l}\text { Preoperative Hb level } \\
(\text { mean }( \pm \text { SD }))\end{array}$ & $8.01(0.92)$ & $7.96(1.02)$ & $7.92(0.91)$ & $0.421^{\wedge}$ \\
\hline $\begin{array}{l}\text { Preoperative GFR (mean } \\
( \pm \text { SD)) }\end{array}$ & $69.0(20.0)$ & $59.0(21.4)$ & $64.4(23.3)$ & $\begin{array}{l}< \\
0.001 "\end{array}$ \\
\hline BMI & & & & $0.434 *$ \\
\hline < 18.5 (obs (\%)) & 20/287 (7.0) & $7 / 100(7.0)$ & $10 / 154(6.5)$ & \\
\hline 18.5-30 (obs (\%)) & 245/287 (85.4) & 79/100 (79.0) & 127/154 (84.5) & \\
\hline > 30 (obs (\%)) & 22/287 (7.6) & $14 / 100(14.0)$ & 17/154 (11.0) & \\
\hline $\begin{array}{l}\text { Time to surgery (median } \\
(\mathrm{IQR}) \text { ) }\end{array}$ & $19.6(13.4-24.6)$ & $24.4(18.5-37.0)$ & $18.8(13.1-24.8)$ & $\begin{array}{l}< \\
0.001 "\end{array}$ \\
\hline Anesthesia & & & & $0.350 *$ \\
\hline Spinal (obs (\%)) & $437 / 490(89.2)$ & 125/142 (88.0) & 180/211 (85.3) & \\
\hline General (obs (\%)) & $53 / 490(10.8)$ & $17 / 142(12.0)$ & $31 / 211(14.7)$ & \\
\hline \multicolumn{5}{|c|}{$\begin{array}{l}\text { Notes: *Pearson's Chi-squared test, }{ }^{\circledR} \text { Student's t-test, }{ }^{*} \text { Wilcoxon rank-sum test, “Kurskal-Wallis, }{ }^{\wedge} \text { linear } \\
\text { regression }\end{array}$} \\
\hline $\begin{array}{l}\text { Abbreviations: NHFS. No } \\
\text { filtration rate in } \mathrm{ml} / \mathrm{min} / 1\end{array}$ & ham Hip Fractu & re; Hb. Hemoglol & $\begin{array}{l}\text { mmol/L; GFR. glc } \\
\text { gy; BMI. Body Ma }\end{array}$ & $\begin{array}{l}\text { ular } \\
\text { idex }\end{array}$ \\
\hline
\end{tabular}

\section{Patients using antiplatelet therapy compared to no anticoagulation}

Patients using AP were significantly older than patients who did not use anticoagulantion (84 years versus 82 years, $P=0.03$ ). Also, less female patients were found in the AP group compared to no anticoagulation use $(132(62 \%)$ versus $355(72 \%), P=0.007)$. Higher NHFS score was found in the AP 
group (5 (4-6) versus 5 (4-6), $P=0.009$ ), and high ASA score (ASA score $\geq 3$ ) was found in the AP group (128 patients $(61 \%)$ versus 224 patients $(46 \%), P=<0.001)$.

\section{Patients using vitamin K antagonists compared to no anticoagulation}

When VKA use was compared to no anticoagulation, higher ASA score (ASA score $\geq 3$ ) was found (106 patients $(75 \%)$ versus 224 patients $(46 \%), P=<0.001)$. Also, prolonged time to surgery was found when VKA was compared to no anticoagulation use, 24.4 hours $(18.5-37.0)$ versus 19.6 hours (13.4-24.6), $\mathrm{P}=$ $<0.001$.

\section{Patients using vitamin K antagonists compared to antiplatelet therapy}

Prolonged time to surgery was found when vitamin K was compared to AP (24.4 hours (18.5-37.0) versus 18.8 (13.1-24.8), $\mathrm{P}=<0.001$, respectively).

\section{International normalized ratio}

At admission, mean INR for patients using vitamin $\mathrm{K}$ antagonists was 3.19 ( \pm 1.71$)$. The mean preoperative INR after correction was $1.48( \pm 0.25)$. Correction was performed using vitamin $\mathrm{K}$ in $55 \%$ of the patients and in $9 \%$ of the patients additional prothrombin complex concentrate was necessary besides vitamin $\mathrm{K}$. In $8 \%$ of the patients receiving correction of the INR, only a prothrombin complex concentrate was used. $22 \%$ of the patients using vitamin $\mathrm{K}$ antagonists received no correction. Of these patients, 12 had an INR $\leq 1.5(1.2( \pm 0.2))$ and eight-teen patients had an INR > $1.5(2.1( \pm 0.4))$. No complications occurred in the last group. Two patients with an INR $>1.5$ without correction received general anesthesia.

\section{Outcome measures}

Univariable analysis was performed on perioperative variables as displayed in Table 3. No difference was found in the 30 -day mortality ( $9 \%$ without anticoagulation versus $10 \%$ with anticoagulation, $P=0.620$ ) nor the 1-year mortality between the groups who did and who did not use anticoagulation drugs ( $26 \%$ versus $29 \%$ respectively, $\mathrm{P}=0.287$ ). 
Table 3

Univariable analysis no anticoagulation use versus anticoagulation use

\begin{tabular}{|c|c|c|c|c|}
\hline Factor & $\begin{array}{l}\text { Overall } \\
\text { (907) }\end{array}$ & $\begin{array}{l}\text { No } \\
\text { anticoagulation } \\
\text { (492) }\end{array}$ & $\begin{array}{l}\text { Anticoagulation } \\
\text { (415) }\end{array}$ & $\begin{array}{l}P- \\
\text { value }\end{array}$ \\
\hline Surgery time (min) & & & & $0.664^{*}$ \\
\hline$<45 \min ($ obs $(\%))$ & $\begin{array}{l}132 / 903 \\
(14.6)\end{array}$ & 75/490 (15.3) & $57 / 413(13.8)$ & \\
\hline$\underset{(\%))}{\geq 45 \min ,<90 \mathrm{~min} \text { (reference) (obs }}$ & $\begin{array}{l}665 / 903 \\
(73.7)\end{array}$ & $361 / 490(73.7)$ & $304 / 413(73.6)$ & \\
\hline$\geq 90 \min ($ obs $(\%))$ & $\begin{array}{l}106 / 903 \\
(11.7)\end{array}$ & $54 / 490(11.0)$ & $52 / 413(12.6)$ & \\
\hline $\begin{array}{l}\text { Minimally invasive surgical } \\
\text { approach (obs (\%)) }\end{array}$ & $\begin{array}{l}223 / 906 \\
(24.6)\end{array}$ & $120 / 491(24.4)$ & $103 / 415(24.8)$ & $0.895^{*}$ \\
\hline Hb loss (mmol/L) (mean \pm SD) & $1.59(0.87)$ & $1.53(0.80)$ & $1.64(0.93)$ & $0.053^{*}$ \\
\hline $\begin{array}{l}\text { Estimated blood loss }(\mathrm{mL}) \text { (mean } \pm \\
\text { SD) }\end{array}$ & $\begin{array}{l}238.2 \\
(143.8)\end{array}$ & $237.9(136.6)$ & $237.1(144.9)$ & $0.738^{*}$ \\
\hline $\begin{array}{l}\text { Packed cell supplementation } \\
\text { (units) (mean } \pm S D \text { ) }\end{array}$ & $0.23(0.75)$ & $0.15(0.61)$ & $0.33(0.88)$ & $\begin{array}{l}< \\
0.001^{*}\end{array}$ \\
\hline Pain day 1 (VAS) (mean \pm SD) & $2.40(2.16)$ & $2.24(2.01)$ & $2.60(2.27)$ & $0.030^{¥}$ \\
\hline Postoperative drain (obs (\%)) & $\begin{array}{l}544 / 900 \\
(60.4)\end{array}$ & $300 / 488(61.5)$ & 244/412 (59.2) & $0.491 *$ \\
\hline Haematoma (obs (\%)) & $\begin{array}{l}122 / 803 \\
(15.2)\end{array}$ & 49/429 (11.4) & 73/374 (19.5) & $0.001 *$ \\
\hline $\begin{array}{l}\text { Reoperation for haematoma (obs } \\
(\%))\end{array}$ & $\begin{array}{l}11 / 888 \\
(1.2)\end{array}$ & $6 / 484(1.2)$ & $5 / 404(1.2)$ & $0.998 *$ \\
\hline Reoperation for luxation (obs (\%)) & $\begin{array}{l}18 / 888 \\
(2.0)\end{array}$ & 9/484 (1.9) & $9 / 404(2.2)$ & $0.698 *$ \\
\hline Deep SSI (obs (\%)) & $\begin{array}{l}44 / 883 \\
(5.0)\end{array}$ & $26 / 481(5.4)$ & $18 / 402(4.5)$ & $0.528 *$ \\
\hline $\begin{array}{l}\text { Ready for discharge (days) } \\
\text { (median (IQR)) }\end{array}$ & $5(3-7)$ & $4(3-7)$ & $5(3-8)$ & $0.006^{*}$ \\
\hline Re-admission (obs (\%)) & $\begin{array}{l}83 / 879 \\
(9.4)\end{array}$ & $46 / 479(9.6)$ & $37 / 400(9.3)$ & $0.858 *$ \\
\hline \multicolumn{5}{|l|}{ Mortality } \\
\hline 30-day mortality & $\begin{array}{l}87 / 907 \\
(9.6)\end{array}$ & $45 / 492(9.2)$ & $42 / 415(10.1)$ & $0.620 *$ \\
\hline
\end{tabular}




\begin{tabular}{|lllll|}
\hline Factor & $\begin{array}{l}\text { Overall } \\
\mathbf{( 9 0 7 )}\end{array}$ & $\begin{array}{l}\text { No } \\
\text { anticoagulation } \\
\mathbf{( 4 9 2 )}\end{array}$ & $\begin{array}{c}\text { Anticoagulation } \\
\mathbf{( 4 1 5 )}\end{array}$ & $\begin{array}{l}\text { P- } \\
\text { value }\end{array}$ \\
\hline One-year mortality & $\begin{array}{l}251 / 907 \\
(27.7)\end{array}$ & $129 / 492(26.2)$ & $122 / 415(29.4)$ & $0.287 *$ \\
\hline Notes: *Pearson's Chi-squared test, ${ }^{*}$ Wilcoxon rank-sum test, & \\
Abbrevations: Hb. Haemoglobin in mmol/L; VAS. Visual Analogue Scale; SSI. Surgical Site Infection. \\
Anticoagulation therapy is vitamin K antagonists and antiplatelet therapy combined.
\end{tabular}

More packed cells were supplemented in patients using anticoagulation compared to patients without anticoagulation, $0.33( \pm 0.88)$ units versus $0.15( \pm 0.61)$ units, respectively. Hematoma's were reported more frequently in patients with anticoagulation use compared to patients without anticoagulation, $20 \%$ vs. $11 \%, P=0.001$, respectively. Also more pain was reported in patients with anticoagulation with a reported VAS of $2.60( \pm 2.27)$ versus $2.24( \pm 2.01) \mathrm{P}=0.030$, one day post-surgery. There was no statistically significant difference in the frequency of post-surgery placed wound drains.

Subgroup analysis are displayed in Table 4. Both vitamin $\mathrm{K}$ antagonists and antiplatelet therapy were associated with more packed cell supplementation compared to no anticoagulation use ( 0.44 units versus 0.15 units, $P=<0.001$, and 0.26 units versus 0.15 units, $P=0.026$ respectively). Also, the use of vitamin $\mathrm{K}$ antagonists was associated with a higher incidence of hematomas compared to no anticoagulation ( $23 \%$ versus $11 \%, P=0.001)$. No association for the occurrence of hematoma was found between the other subgroups. At last, patients who used vitamin $\mathrm{K}$ antagonists needed more days to be ready for discharge compared to no anticoagulation, 5.5 days (4-9) versus 4 days (3-7), $P=<0.001$. 
Table 4

Univariable subgroup analysis

$\begin{array}{lllll}\text { Factor } & \begin{array}{l}\text { No } \\ \text { anticoagulation } \\ \text { (492) }\end{array} & \begin{array}{l}\text { Vitamin K } \\ \text { antagonist (141) }\end{array} & \begin{array}{l}\text { Antiplatelet } \\ \text { therapy (213) }\end{array} & \begin{array}{l}\text { P- } \\ \text { value* }\end{array} \\ & & & \end{array}$

\section{Surgery time (min)}

$<45 \min ($ obs (\%))

$\geq 45 \mathrm{~min},<90 \mathrm{~min}$ (reference) (obs (\%))

$\geq 90 \mathrm{~min}$ (obs (\%))

Minimally invasive surgical approach (obs (\%))

$\mathrm{Hb}$ loss (mmol/l) (mean $\pm \mathrm{SD})$

Estimated blood loss ( $\mathrm{mL}$ ) (mean \pm SD)

Packed cell supplementation
(units) (mean $\pm S D$ )

Pain day 1 (VAS) (mean \pm SD)

Postoperative drain (obs (\%))

Haematoma (obs (\%))

Reoperation for haematoma (obs (\%))

Reoperation for luxation (obs $(\%)$ )

Deep SSI (obs (\%))

Ready for discharge (days) (median (IQR))

Re-admission (obs (\%))

Mortality

30-day mortality

One-year mortality

Notes: *Pearson's Chi-squared test, ${ }^{\circledR}$ Student's t-test, ${ }^{*}$ Wilcoxon rank-sum test, “Kurskal-Wallis, ${ }^{\wedge}$ linear regression

Abbreviations: Abbrevations: Hb. Haemoglobin in mmol/L; VAS. Visual Analogue Scale; SSI. Surgical Site Infection.

\begin{tabular}{lll}
$75 / 490(15.3)$ & $22 / 141(15.6)$ & $26 / 212(15.6)$ \\
\hline $361 / 490(73.7)$ & $100 / 141(70.9)$ & $163 / 212(76.9)$
\end{tabular}

$54 / 490(11.0) \quad 19 / 141(13.5)$

23/212 (10.9)

$120 / 491(24.4) \quad 43 / 142(30.3)$

$47 / 213(22.1)$

$0.205^{*}$

$1.53(0.80)$

$1.59(1.05)$

$1.62(0.9)$

$0.323^{\prime \prime}$

$237.9(136.6) \quad 242.8(149.4)$

$235.3(136.8)$

$0.946^{\prime \prime}$

$0.15(0.6)$

$0.44(1.1)$

$0.26(0.8)$

$<$

$0.001^{\prime \prime}$

$2.24(2.0)$

$2.62(2.4)$

$2.56(2.2)$

0.158

$300 / 488(61.5)$

83/142 (58.5)

$129 / 210(61.4)$

$0.799 *$ $49 / 429$ (11.4)

$30 / 130(23.1)$

$30 / 188(16.0)$

$0.004^{*}$

$6 / 484(1.2)$

$3 / 136(2.2)$

$1 / 208(0.5)$

$0.357 *$

$9 / 484(1.9)$

$2 / 136(1.5)$

$7 / 208(3.4)$

$0.381^{\star}$

26/481 (5.4)

$7 / 135(5.2)$

8/207 (3.9)

0.691 *

$4(3-7)$

$5.5(4-9)$

$4(3-7)$

$0.001^{\prime \prime}$

$46 / 479(9.6)$

$18 / 136(13.2)$

$15 / 204$

$0.198 *$

$\begin{array}{llll}45 / 492(9.2) & 18 / 142(12.7) & 20 / 213(9.4) & 0.448^{*} \\ 129 / 492(26.2) & 47 / 142(33.1) & 59 / 213(27.7) & 0.272^{*}\end{array}$

129/492 (26.2) $\quad 47 / 142(33.1)$

$59 / 213(27.7)$

$0.272^{*}$ 


\section{Clopidogrel (Plavix)}

Twenty-three patients used clopidogrel (Plavix). The median age of these patients was 85 years (78-90), and $74 \%$ was female. A median NHFS of 5 (4-6) was found, and $78 \%$ of the patients had an ASA score 23. A mean preoperative hemoglobin level of 7.9 (7.4-8.4) was found, and a mean preoperative GFR of $72(56-85)$. For BMI, $83 \%$ of the patients had a BMI between 18.5 and 30 . Time to surgery showed a median of 22.8 hours $(17.8-26.9)$ and most patients $(70 \%)$ received spinal anesthesia. For clopidogrel the mean $\mathrm{Hb}$ loss was $1.5 \mathrm{mmol} / \mathrm{L}(1-2.2)$. The estimated blood loss was $238 \mathrm{~mL}( \pm 143)$ and the number of units of packed cells supplemented was $0.23( \pm 0.75)$.

\section{Discussion}

This current study describes the effect of traditional anticoagulation therapy on surgical treatment of geriatric patients with a femoral neck fracture for which a hip hemiarthroplasty was placed.

The finding with the most extensive clinical impact was a prolonged time to surgery in patients using vitamin $\mathrm{K}$ antagonists, compared to patients without anticoagulation ( 24.4 hours versus 19.6 hours, $\mathrm{P}=<$ 0.001). This delay in time to surgery was found despite correction of INR with either vitamin $K$, prothrombin complex concentrate or a combination of both. In accordance to current literature, vitamin $\mathrm{K}$ was used in $55 \%$ of the patients in our study using a vitamin $\mathrm{K}$ antagonist for correction of the INR. ${ }^{(1)(7)}$ The delay in time to surgery was previously found by Lawrence et al. in 2016 and Tran et al. in 2015, who found that after controlling for baseline variables, vitamin $\mathrm{K}$ antagonists were associated with increased

time to surgery. ${ }^{(5)(14)}$ One explanation for prolonged time to surgery is the previously mentioned required correction of international normalized ratio. An INR $<1.5$ during surgery is advised to perform spinal anesthesia and surgery as safe as possible. ${ }^{(15)}$ Despite adequate correction of INR, hematomas were found more frequently in patients using anticoagulation. After subgroup analysis, hematomas most frequently occurred in the vitamin $\mathrm{K}$ antagonist subgroup ( $23.1 \%$ versus $11.4 \%$ without anticoagulation use, $P=0.001$ ). No difference was found when antiplatelet therapy was compared to no anticoagulation use. One explanation of the increased risk for hematoma in patients using vitamin $\mathrm{K}$ antagonists, is the short half-life of vitamin $\mathrm{K}$ as reversal therapy for vitamin $\mathrm{K}$ antagonists, which is 24.7 hours, and therefore a rebound effect in the postoperative period can occur. ${ }^{(6)}$ Since hematomas show significant association with deep surgical site infections, precautions should be taken to prevent these hematomas from developing. Adequate correction of INR is one of those precautions. ${ }^{(12)}$

Also, patients using vitamin $\mathrm{K}$ antagonists needed more days to be ready for discharge compared to antiplatelet therapy as well as compared to patients without anticoagulation (5.5 (4-9) days versus 4 (37 ) days, $P=0.002$, and 5.5 (4-9) days versus $4(3-7)$ days, $p<0.001$, respectively). No differences were found in the use of wound drains and the days until a drain was removed, as explanation for the prolonged time to be ready for discharge. 
One variable that could be the explanation for this finding is the administration of more packed cells postoperative in patients using vitamin $\mathrm{K}$ antagonists. Anemia and the administration of packet cells could have prolonged hospital stay. Patients who used anticoagulation were in need of more packed cell supplementation than patients without anticoagulation, respectively $0.33( \pm 0.88)$ versus $0.15( \pm 0.61)$ packed cells, $\mathrm{P}=<0.001$. For subgroup analysis, the use of both vitamin $\mathrm{K}$ antagonists and antiplatelet therapy leads to more packed cell supplementation compared to no anticoagulation $(0.44( \pm 1.1)$ versus $0.15( \pm 0.6), \mathrm{p}<0.001$ for vitamin $\mathrm{K}$ antagonists versus no anticoagulation and $0.26( \pm 0.8)$ versus $0.15( \pm$ $0.6), P=0.026$ for antiplatelet therapy versus no anticoagulation). In accordance to our findings Cha et al. in 2018 also found that even thrombocyte aggregation inhibitors, such as aspirin, were associated with more packed cell supplementation. ${ }^{(16)}$ This is possible due to the duration of the effect of thrombocyte aggregation inhibitors, which last during the entire lifespan of the platelets, which is usually 5 to 9 days. (17) Since prolonged hospital stay is associated with complications such as pneumonias and decubital ulcers, a stay as short as possible is desirable for these fragile patients. ${ }^{(1)}$

\section{Strengths and limitations}

One of the limitations of this study is the potential absence of data on confounding factors since baseline characteristics and clinical data had to be completed retrospectively. However, the prospective hip fracture databased used in this study tried to prevent selection bias and absence of data regarding most variables. Since standard of care in our hospital is to correct INR in advance to surgery to an INR $\leq$ 1.5 , the presented data here could be an underestimation of the clinical outcomes if no correction for INR had been performed. Previously published articles are limited by small sample sizes and heterogeneous outcomes. Our study provides large and comparable groups in oral anticoagulants versus no anticoagulation use, with subgroup analysis per type oral anticoagulant.

\section{Conclusion}

This present study shows that patients using anticoagulation are in need of more packed cell supplementation, develop more hematomas and need more days to be ready for discharge, compared to patients without anticoagulation use. Also a prolonged time to surgery was found for patients using vitamin K antagonists, despite correction of INR. Quick and aggressive correction of INR should be one of the main points of attention to prevent the still present prolonged time to surgery in day to day practice. One of the main goals in treating a patient with a femoral neck fracture should be performing surgery as soon as possible after developing the fracture, to anticipate to preventable complications. We however believe that the use of any kind of anticoagulation medication should be an indicator for frailty of patients, and therefore careful considerations should be made between the timing of surgery and the expected effect of anticoagulation on clinical outcomes.

\section{List Of Abbreviations}

AP Antiplatelet therapy 
ASA American Society of Anesthesiologist

dL Deciliter

g Gram

INR International Normalized Ratio

IQR Interquartile Range

$\mathrm{mL}$ Milliliter

NHFS Nottingham Hip Fracture Score

SD Standard Deviation

SSI Surgical Site Infection

VAS Visual Analogue Scale

VKA Vitamin K Antagonists

\section{Declarations}

\section{Ethics approval and consent to participate:}

The local ethics committee (Toetsingscommissie Wetenschappelijk Onderzoek Rotterdam (TWOR)) has approved the study protocol (study number L2017044). The same committee also concluded that due to the high percentage of cognitive dysfunction in the study population, and since there were no changes in standard practice of care, patients' consent to review their medical records was not required and was therefore waived. All protocols were in compliance with the declaration of Helsinki and patient data was stored anonymously.

\section{Consent for publication:}

not applicable.

\section{Availability of data and material:}

data is available in encrypted files and will only be provided on reasonable request due to ongoing inclusion in this study. Data can be requested by contacting the corresponding author.

\section{Conflicts of interest/Competing interests:}

nothing to declare 


\section{Funding:}

no funding was received for this study

\section{Authors' contributions:}

VvR study coordinator, data analysis, writing the paper, LdJ data analysis, writing the paper, TMALK writing the paper, TMK data analysis, writing the paper, GR writing the paper.

\section{Acknowledgements:}

not applicable

\section{References}

1. Buecking B, Eschbach D, Bliemel C, Oberkircher L, Struewer J, Ruchholtz S, et al. Effectiveness of vitamin $\mathrm{K}$ in anticoagulation reversal for hip fracture surgery-a prospective observational study. Thrombosis research. 2014;133(1):42-7.

2. Daugaard C, Pedersen AB, Kristensen NR, Johnsen SP. Preoperative antithrombotic therapy and risk of blood transfusion and mortality following hip fracture surgery: a Danish nationwide cohort study. Osteoporos Int Osteoporosis International, 2019;30(3):583-591.

3. Smith EB, Parvizi J, Purtill JJ. Delayed surgery for patients with femur and hip fractures-risk of deep venous thrombosis. The Journal of trauma. 2011;70(6):E113-6.

4. Maxwell MJ, Moran CG, Moppett IK. Development and validation of a preoperative scoring system to predict 30 day mortality in patients undergoing hip fracture surgery. British journal of anaesthesia. 2008;101(4):511-7.

5. Tran T, Delluc A, de Wit C, Petrcich W, Le Gal G, Carrier M. The impact of oral anticoagulation on time to surgery in patients hospitalized with hip fracture. Thrombosis research. 2015;136(5):962-5.

6. Combettes E, Mazoit JX, Benhamou D, Beloeil H. Modelling of vitamin K half-life in patients treated with vitamin $\mathrm{K}$ antagonists before hip fracture surgery. Anaesthesia, critical care \& pain medicine. 2015;34(5):295-9.

7. Levy JH, Douketis J, Weitz Jl. Reversal Agents for Non-Vitamin K Antagonist Oral Anticoagulants. Nat Rev Cardiol. 2018 May; 15(5):273-281

8. Collinge CA, Kelly KC, Little B, Weaver T, Schuster RD. The effects of clopidogrel (Plavix) and other oral anticoagulants on early hip fracture surgery. Journal of orthopaedic trauma. 2012;26(10):56873.

9. Ginsel BL, Taher A, Whitehouse SL, Bell JJ, Pulle CR, Crawford RW. Effects of anticoagulants on outcome of femoral neck fracture surgery. Journal of orthopaedic surgery (Hong Kong). 2015;23(1):29-32. 
10. Doleman B, Moppett IK. Is early hip fracture surgery safe for patients on clopidogrel? Systematic review, meta-analysis and meta-regression. Injury. 2015;46(6):954-62.

11. Douketis JD, Spyropoulos AC, Spencer FA. Perioperative management of antithrombotic therapy: Antithrombotic Therapy and Prevention of Thrombosis, 9th ed: American Colege of Chest Physicians Evidence-Based Clinical Practice Guidelines. Chest 2012; 141:e326S-50S.

12. de Jong L, Klem TMAL, Kuiper TM. Factors affecting the rate of surgical site infection in patients after hemiarthroplasty of the hip following a fracture of the neck of the femur. Bone Joint J 2017;99B:1088-94

13. Maxwell MJ, Moran CG, Moppett IK. Development and validation of a preoperative scoring system to predict 30 day mortality in patients undergoing hip fracture surgery. $\mathrm{Br} \mathrm{J}$ Anaesth 2008;101:511-7.

14. Lawrence JE, Fountain DM, Cundall-Curry DJ, Carrothers AD. Do Patients Taking Warfarin Experience Delays to Theatre, Longer Hospital Stay, and Poorer Survival After Hip Fracture? Clinical orthopaedics and related research. 2017;475(1):273-9.

15. Cohn MR, Levack AE, Trivedi NN, Villa JC, Wellman DS, Lyden JP, et al. The Hip Fracture Patient on Warfarin: Evaluating Blood Loss and Time to Surgery. Journal of orthopaedic trauma. 2017;31(8):407-13.

16. Cha YH, Lee YK, Koo KH, Wi C, Lee KH. Difference in Mortality Rate by Type of Anticoagulant in Elderly Patients with Cardiovascular Disease after Hip Fractures. Clinics in orthopedic surgery. 2019;11(1):15-20.

17. Gleason LJ, Friedman SM. Preoperative management of anticoagulation and antiplatelet agents. Clinics in geriatric medicine. 2014;30(2):219-27.

\section{Figures}




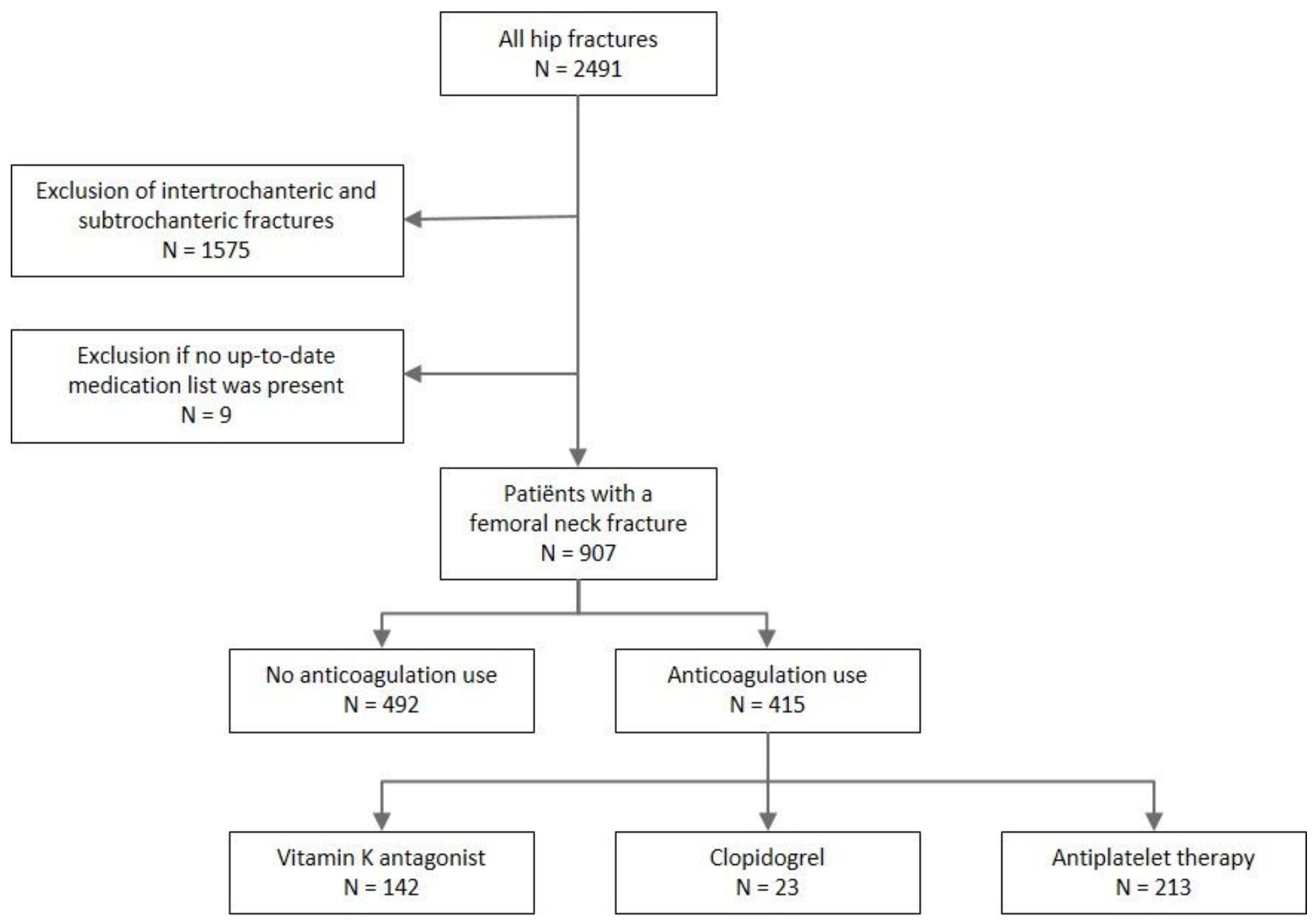

\section{Figure 1}

The inclusion flowchart 